\title{
TIME AVERAGING FOR FUNCTIONAL DIFFERENTIAL EQUATIONS
}

\section{MUSTAPHA LAKRIB}

Received 14 March 2002 and in revised form 1 August 2002

We present a result on the averaging for functional differential equations on finite time intervals. The result is formulated in both classical mathematics and nonstandard analysis; its proof uses some methods of nonstandard analysis.

\section{Introduction}

The idea of the method of averaging is to determine conditions in which solutions of an autonomous dynamical system can be used to approximate solutions of a more complicated time varying dynamical system. The method of averaging has become one of the most important tool ever developed for nonlinear time varying systems. Applications have been found in celestial mechanics, noise control, nonlinear oscillations, stability analysis, bifurcation theory and vibrational control, among many other fields. Although averaging of ordinary differential equations is considered a mature field-the reader may consult $[1,5,8,22,24]$ for more references and information on the subject (see also [13, 14]) - averaging of functional differential equations has only recently been developed (see $[6,7,9,11,12,15,16,19]$ ).

This paper aims to present a result on the averaging for functional differential equations of the form

$$
\dot{x}(t)=f\left(\frac{t}{\varepsilon}, x_{t}\right)
$$

Copyright @ 2003 Hindawi Publishing Corporation

Journal of Applied Mathematics 2003:1 (2003) 1-16

2000 Mathematics Subject Classification: 34C29, 34K25, $03 \mathrm{H} 05$

URL: http:/ /dx.doi.org/10.1155/S1110757X03203077 
2 Time averaging for functional differential equations

on finite time intervals. The result is not new (see [10] and the references therein). However, by means of nonstandard analysis methods, we propose a new proof where all the analysis is achieved in $\mathbb{R}^{d}$ (it is not the case in [10]) which makes it more simple.

The paper is organized as follows. Section 2 contains the notation and conditions required to state and prove our main result as well as the main result itself. The proof of this result is given in Section 4.2. To avoid complicating the proof unnecessarily, several subsidiary lemmas have been placed in Section 4.1.

The main result is formulated in both classical mathematics and nonstandard analysis. Its proof makes use of Robinson's nonstandard analysis (NSA) [21]. We will work in the axiomatic form IST (for internal set theory) of nonstandard analysis, given by Nelson [20]. For that, Section 3.1 is devoted to a short description of IST. Then, in Section 3.2, we present the nonstandard translate (Theorem 3.6) in the language of IST of our main result (Theorem 2.2). We recall that IST is a conservative extension of ordinary mathematics. This means that any statement of ordinary mathematics which is a theorem of IST was already a theorem of ordinary mathematics, so there is no need to translate the proof.

\section{Notation, conditions, and main result}

Let $r \geq 0$ be a given constant. Throughout this paper $\mathcal{C}_{0}=\mathcal{C}\left([-r, 0], \mathbb{R}^{d}\right)$ will denote the Banach space of all continuous functions from $[-r, 0]$ into $\mathbb{R}^{d}$ with the norm $\|\phi\|=\sup \{|\phi(\theta)|:-r \leq \theta \leq 0\}$, where $|\cdot|$ is a norm of $\mathbb{R}^{d}$. Let $t_{0} \in \mathbb{R}$ and $T>t_{0}$. If $x(t)$ is a continuous function defined on $\left[t_{0}-r, T\right]$ and $t \in\left[t_{0}, T\right]$, then $x_{t} \in \mathcal{C}_{0}$ is defined by $x_{t}(\theta)=x(t+\theta)$ for $\theta \in[-r, 0]$.

The hypotheses, which are denoted by the letter $\mathrm{H}$, are listed as follows.

(H1) The functional $f: \mathbb{R} \times \mathcal{C}_{0} \rightarrow \mathbb{R}^{d}$ in (1.1) is continuous.

(H2) The functional $f$ is Lipschitzian in $u \in \mathcal{C}_{0}$, that is, there exists some constant $k$ such that

$$
\left|f\left(\tau, u_{1}\right)-f\left(\tau, u_{2}\right)\right| \leq k\left\|u_{1}-u_{2}\right\|, \quad \forall \tau \in \mathbb{R}, \forall u_{1}, u_{2} \in \mathcal{C}_{0} .
$$

(H3) For all $u \in \mathcal{C}_{0}$, there exists a limit

$$
f^{0}(u):=\lim _{T \rightarrow \infty} \frac{1}{T} \int_{0}^{T} f(\tau, u) d \tau .
$$


For any $\phi \in \mathcal{C}_{0}$ and $t_{0} \in \mathbb{R}$, the solution of the averaged equation

$$
\dot{y}(t)=f^{0}\left(y_{t}\right)
$$

(resp., the solution of (1.1)) such that $y_{t_{0}}=\phi$ (resp., $x_{t_{0}}=\phi$ ) is denoted by $y=y\left(\cdot ; t_{0}, \phi\right)$ (resp., $\left.x=x\left(\cdot ; t_{0}, \phi\right)\right)$ and $J($ resp., $I)$ will denote its maximal interval of definition.

Remark 2.1. Existence and uniqueness of solutions of (2.3) will be justified a posteriori. Indeed, we will show in Lemma 4.1 below that the function $f^{0}$ is $k$-Lipschitz so that existence and uniqueness are guaranteed.

Under the above assumptions, we will state the main result of this paper which gives nearness of solutions of (1.1) and (2.3) on finite time intervals.

THEOREM 2.2. Let assumptions (H1), (H2), and (H3) hold. Let $\phi \in \mathcal{C}_{0}$ and $t_{0} \in$ $\mathbb{R}$. Let $x$ be the solution of (1.1) and $y$ the solution of (2.3) with $x_{t_{0}}=y_{t_{0}}=\phi$. Then for any $\delta>0$ and $T>t_{0}, T \in J$, there exists $\varepsilon_{0}=\varepsilon_{0}(\delta, T)>0$ such that, for $\varepsilon \in\left(0, \varepsilon_{0}\right], x$ is defined at least on $\left[t_{0}, T\right]$ and $|x(t)-y(t)|<\delta$ on $t \in\left[t_{0}, T\right]$.

\section{Nonstandard main result}

\subsection{Internal set theory}

In IST we adjoin to ordinary mathematics (say ZFC) a new undefined unary predicate standard (st). The axioms of IST are the usual axioms of ZFC plus three others which govern the use of the new predicate. Hence, all theorems of ZFC remain valid in IST. What is new in IST is an addition, not a change. We call a formula of IST external in the case where it involves the new predicate st; otherwise, we call it internal. Thus internal formulas are the formulas of ZFC. The theory IST is a conservative extension of ZFC, that is, every internal theorem of IST is a theorem of ZFC. Some of the theorems which are proved in IST are external and can be reformulated so that they become internal. Indeed, there is a reduction algorithm which reduces any external formula $F\left(x_{1}, \ldots, x_{n}\right)$ of IST without other free variables than $x_{1}, \ldots, x_{n}$, to an internal formula $F^{\prime}\left(x_{1}, \ldots, x_{n}\right)$ with the same free variables, such that $F \equiv F^{\prime}$, that is, $F \Leftrightarrow F^{\prime}$ for all standard values of the free variables. In other words, any result which may be formalized within IST by a formula $F\left(x_{1}, \ldots, x_{n}\right)$ is equivalent to the classical property $F^{\prime}\left(x_{1}, \ldots, x_{n}\right)$, provided the parameters $x_{1}, \ldots, x_{n}$ are restricted to standard values. Here is the reduction of the frequently 
4 Time averaging for functional differential equations

occurring formula $\forall x\left(\forall^{\text {st }} y A \Rightarrow \forall^{\text {st }} z B\right)$ where $A$ and $B$ are internal formulas

$$
\forall x\left(\forall^{\text {st }} y A \Longrightarrow \forall^{\text {st }} z B\right) \equiv \forall z \exists^{\mathrm{fin}} y^{\prime} \forall x\left(\forall y \in y^{\prime} A \Longrightarrow B\right)
$$

The notations $\forall^{\text {st }} X$ and $\exists^{\text {fin }} X$ stand for $[\forall X, \operatorname{st}(X) \Rightarrow \cdots]$ and $[\exists X$, $X$ finite \&...], respectively.

A real number $x$ is called infinitesimal when $|x|<a$ for all standard $a>0$, limited when $|x| \leq a$ for some standard $a$, appreciable when it is limited and not infinitesimal, and unlimited, when it is not limited. We use the following notations: $x \simeq 0$ for $x$ infinitesimal, $x \simeq+\infty$ for $x$ unlimited positive, $x \gg 0$ for $x$ noninfinitesimal positive. Thus we have

$$
\begin{aligned}
x \simeq 0 & \Longleftrightarrow \forall^{\text {st }} a>0|x|<a, \\
x \gg 0 & \Longleftrightarrow \exists^{\text {st }} a>0 x \geq a, \\
x \text { limited } & \Longleftrightarrow \exists^{\text {st }} a>0|x| \leq a, \\
x \simeq+\infty & \Longleftrightarrow \forall^{\text {st }} a>0 x>a .
\end{aligned}
$$

Let $(E, d)$ be a standard metric space. Two points $x$ and $y$ in $E$ are called infinitely close, denoted by $x \simeq y$, when $d(x, y) \simeq 0$. If there exists in that space a standard $x_{0}$ such that $x \simeq x_{0}$, the element $x$ is called nearstandard in $E$ and the standard point $x_{0}$ is called the standard part of $x$ (it is unique) and is also denoted by ${ }^{o} x$. A vector in $\mathbb{R}^{d}$ ( $d$ standard) is said to be infinitesimal (resp., limited) if its norm $|x|$ is infinitesimal (resp., limited), where $|\cdot|$ is a norm in $\mathbb{R}^{d}$.

We may not use external formulas to define subsets. The notations $\{x \in \mathbb{R}: x$ is limited $\}$ or $\{x \in \mathbb{R}: x \simeq 0\}$ are not allowed. Moreover, we can prove the following lemma.

LeMma 3.1. There do not exist subsets $\mathcal{\perp}$ and $\supset$ of $\mathbb{R}$ such that, for all $x \in \mathbb{R}$, $x$ is in $\mathcal{L}$ if and only if $x$ is limited, or $x$ is in $\supset$ if and only if $x$ is infinitesimal.

It happens sometimes in classical mathematics that a property is assumed, or proved, on a certain domain, and that afterwards it is noticed that the character of the property and the nature of the domain are incompatible. So actually the property must be valid on a larger domain. In the same manner, in nonstandard analysis, the result of Lemma 3.1 is frequently used to prove that the validity of a property exceeds the domain where it was established in direct way. Suppose that we have shown that $A$ holds for every limited $x$, then we know that $A$ holds for some unlimited $x$, for otherwise we could let $\mathcal{L}=\{x \in \mathbb{R}: A\}$. This statement is called the Cauchy principle. It has the following consequence. 
LEMMA 3.2 (Robinson's lemma). Let $g$ be a real function such that $g(t) \simeq 0$ for all limited $t \geq 0$, then there exists an unlimited positive number $\omega$ such that $g(t) \simeq 0$ for all $t \in[0, \omega]$.

Proof. The set of all $s$ such that for all $t \in[0, s]$ we have $|g(t)|<1 / s$ contains all limited $s \geq 1$. By the Cauchy principle it must contain some unlimited $\omega$.

We conclude this section with two other applications of the Cauchy principle which will be used later.

LEMMA 3.3. If $D(\cdot)$ is an internal property such that $D(\lambda)$ holds for all appreciable real numbers $\lambda>0$, then there exists $0<\lambda_{0} \simeq 0$ such that $D\left(\lambda_{0}\right)$ holds.

LEMMA 3.4. Let $h: I \rightarrow \mathbb{R}$ be a function such that $h(t) \simeq 0$ for all $t \in I$. Then $\sup \{h(t): t \in I\} \simeq 0$.

Remark 3.5. The use of nonstandard analysis in perturbation theory of differential equations goes back to the seventies with the Reebian school (see $[17,18]$ and the references therein). It gave birth to the nonstandard perturbation theory of differential equations which has become today a well-established tool in asymptotic theory. For more informations on nonstandard analysis and its applications, the reader is referred to $[2,3$, $4,20,21,23]$.

\subsection{Main result: nonstandard formulation}

Hereafter we give the nonstandard formulation of Theorem 2.2. Then, by use of the reduction algorithm, we show that the reduction of Theorem 3.6 is Theorem 2.2.

THEOREM 3.6. Let $f: \mathbb{R} \times \mathcal{C}_{0} \rightarrow \mathbb{R}^{d}$ be standard. Assume that assumptions (H1), (H2), and (H3) hold. Let $\phi \in \mathcal{C}_{0}$ and $t_{0} \in \mathbb{R}$ be standard. Let $x$ be the solution of (1.1) and $y$ the solution of (2.3) with $x_{t_{0}}=y_{t_{0}}=\phi$. Let $\varepsilon>0$ be infinitesimal. Then for any standard $T>t_{0}, T \in J, x$ is defined at least on $\left[t_{0}, T\right]$ and $x(t) \simeq y(t)$ for all $t \in\left[t_{0}, T\right]$.

The proof of Theorem 3.6 is postponed to Section 4 . Theorem 3.6 is an external statement. As we have recalled, Nelson [20] proposed a reduction algorithm that reduces external theorems to equivalent internal forms. We show that the reduction of Theorem 3.6 is Theorem 2.2.

Reduction of Theorem 3.6. Without loss of generality, let $t_{0}=0$. Let $T>$ $0, T \in J$, and $T$ standard. The characterization of the conclusion of 
6 Time averaging for functional differential equations

Theorem 3.6 is

$$
\begin{gathered}
\forall \varepsilon: \varepsilon \simeq 0 \Longrightarrow x \text { is defined at least on }[0, T] \\
\text { and } x(t) \simeq y(t) \text { for all } t \in[0, T] .
\end{gathered}
$$

Let $B$ be the formula "If $\delta>0$ then $x$ is defined at least on $[0, T]$ and $|x(t)-y(t)|<\delta$ on $t \in[0, T]$." Using (3.2), formula (3.3) becomes

$$
\forall \varepsilon\left(\forall^{\mathrm{st}} \eta \varepsilon<\eta \Longrightarrow \forall^{\mathrm{st}} \delta B\right) .
$$

In this formula $T$ is standard and $\varepsilon, \eta$, and $\delta$ range over the strictly positive real numbers. By (3.1), formula (3.4) is equivalent to

$$
\forall \delta \exists^{\text {fin }} \eta^{\prime} \forall \varepsilon\left(\forall \eta \in \eta^{\prime} \varepsilon<\eta \Longrightarrow B\right) .
$$

For $\eta^{\prime}$ a finite set, $\forall \eta \in \eta^{\prime}, \varepsilon<\eta$ is the same as $\varepsilon<\varepsilon_{0}$ for $\varepsilon_{0}=\min \eta^{\prime}$, and so formula (3.5) is equivalent to

$$
\forall \delta \exists \varepsilon_{0} \forall \varepsilon\left(\varepsilon<\varepsilon_{0} \Longrightarrow B\right) .
$$

That is the statement of Theorem 2.2 holds for any standard $T>0, T \in J$. By transfer, it holds for any $T>0, T \in J$.

\section{Proof of Theorem 3.6}

\subsection{Preliminary lemmas}

In this subsection we state some results which are needed for our proof of Theorem 3.6. Let $f: \mathbb{R} \times \mathcal{C}_{0} \rightarrow \mathbb{R}^{d}$ be standard. The external formulations of conditions $(\mathrm{H} 1),(\mathrm{H} 2)$, and $(\mathrm{H} 3)$ are, respectively,

$\left(\mathrm{H} 1^{\prime}\right) \forall^{\text {st }} \tau \in \mathbb{R} \forall^{\text {st }} u \in \mathcal{C}_{0} \forall \tau^{\prime} \in \mathbb{R} \forall u^{\prime} \in \mathcal{C}_{0}$ :

$$
\tau^{\prime} \simeq \tau, u^{\prime} \simeq u \Longrightarrow f\left(\tau, u^{\prime}\right) \simeq f(\tau, u) .
$$

$\left(\mathrm{H}^{\prime}\right)$ There is a standard constant $k$ such that

$$
\left|f\left(\tau, u_{1}\right)-f\left(\tau, u_{2}\right)\right| \leq k\left\|u_{1}-u_{2}\right\|, \quad \forall^{\text {st }} \tau \in \mathbb{R}, \forall^{\text {st }} u_{1}, u_{2} \in \mathcal{C}_{0}
$$

(and by transfer the inequality holds for all $\tau \in \mathbb{R}$ and all $u_{1}, u_{2} \in$ $\left.\mathrm{C}_{0}\right)$.

$\left(\mathrm{H}^{\prime}\right)$ There is a standard functional $f^{0}: \mathcal{C}_{0} \rightarrow \mathbb{R}^{d}$ such that

$$
\forall^{\text {st }} u \in \mathcal{C}_{0}, \forall T \simeq+\infty, \quad f^{0}(u) \simeq \frac{1}{T} \int_{0}^{T} f(\tau, u) d \tau .
$$


We prove the following lemmas.

LEMMA 4.1. The functional $f^{0}$ is Lipschitz (with the same constant of Lipschitz as $f$ ) and satisfies

$$
f^{0}(u) \simeq \frac{1}{T} \int_{0}^{T} f(\tau, u) d \tau
$$

for all $u \in \mathcal{C}_{0}, u$ nearstandard, and all $T \simeq+\infty$.

Proof. First, let $u_{1}, u_{2} \in \mathcal{C}_{0}$, with $u_{1}$ and $u_{2}$ standard. By means of conditions (H2) and (H3), we have

$$
\left|f^{0}\left(u_{1}\right)-f^{0}\left(u_{2}\right)\right| \leq \lim _{T \rightarrow \infty} \frac{1}{T} \int_{0}^{T}\left|f\left(\tau, u_{1}\right)-f\left(\tau, u_{2}\right) d \tau\right| \leq k\left\|u_{1}-u_{2}\right\| .
$$

That is, $f^{0}$ is $k$-Lipschitz.

Next, let $u,{ }^{0} u \in \mathcal{C}_{0}$ such that ${ }^{o} u$ is standard and $u \simeq{ }^{0} u$. By means of $(4.5)$, conditions $\left(\mathrm{H}^{\prime}\right)$ and $\left(\mathrm{H} 2^{\prime}\right)$, respectively, for all $T \simeq+\infty$, we have

$$
f^{0}(u) \simeq f^{0}\left({ }^{0} u\right) \simeq \frac{1}{T} \int_{0}^{T} f\left(\tau,{ }^{0} u\right) d \tau \simeq \frac{1}{T} \int_{0}^{T} f(\tau, u) d \tau .
$$

LEMMA 4.2. There exists $\mu>0$ such that whenever $t \geq 0$ is limited and $u \in \mathcal{C}_{0}$ is nearstandard there exists $\alpha>0$ such that $\mu<\alpha \simeq 0$ and

$$
\frac{\varepsilon}{\alpha} \int_{t / \varepsilon}^{t / \varepsilon+\alpha / \varepsilon} f(\tau, u) d \tau \simeq f^{0}(u) .
$$

Proof. Let $t \geq 0$ be limited and let $u \in \mathcal{C}_{0}$ be nearstandard.

Case 1 ( $t$ is such that $t / \varepsilon$ is limited). Let $S>0$ be unlimited such that $\varepsilon S \simeq 0$. Then

$$
\frac{1}{S} \int_{t / \varepsilon}^{t / \varepsilon+S} f(\tau, u) d \tau=\left(1+\frac{t}{\varepsilon S}\right) \frac{1}{t / \varepsilon+S} \int_{0}^{t / \varepsilon+S} f(\tau, u) d \tau-\frac{1}{S} \int_{0}^{t / \varepsilon} f(\tau, u) d \tau .
$$

By Lemma 4.1 we have

$$
\frac{1}{t / \varepsilon+S} \int_{0}^{t / \varepsilon+S} f(\tau, u) d \tau \simeq f^{0}(u)
$$

Since

$$
\frac{1}{S} \int_{0}^{t / \varepsilon} f(\tau, u) d \tau \simeq 0, \quad \frac{t}{\varepsilon S} \simeq 0
$$


8 Time averaging for functional differential equations

we have

$$
\frac{1}{S} \int_{t / \varepsilon}^{t / \varepsilon+S} f(\tau, u) d \tau \simeq f^{0}(u)
$$

Then, it suffices to choose $\mu=\varepsilon$ and take $\alpha=\varepsilon S$.

Case 2 ( $t$ is such that $t / \varepsilon$ is unlimited). Let $S>0$. We write

$$
\begin{aligned}
\frac{1}{S} \int_{t / \varepsilon}^{t / \varepsilon+S} f(\tau, u) d \tau= & \frac{1}{t / \varepsilon+S} \int_{0}^{t / \varepsilon+S} f(\tau, u) d \tau \\
& +\frac{t}{\varepsilon S}\left(\frac{1}{t / \varepsilon+S} \int_{0}^{t / \varepsilon+S} f(\tau, u) d \tau-\frac{1}{t / \varepsilon} \int_{0}^{t / \varepsilon} f(\tau, u) d \tau\right) .
\end{aligned}
$$

By Lemma 4.1 we have

$$
\frac{1}{t / \varepsilon+S} \int_{0}^{t / \varepsilon+S} f(\tau, u) d \tau \simeq f^{0}(u) \simeq \frac{1}{t / \varepsilon} \int_{0}^{t / \varepsilon} f(\tau, u) d \tau .
$$

We denote

$$
\eta(S)=\frac{t}{\varepsilon S}\left(\frac{1}{t / \varepsilon+S} \int_{0}^{t / \varepsilon+S} f(\tau, u) d \tau-\frac{1}{t / \varepsilon} \int_{0}^{t / \varepsilon} f(\tau, u) d \tau\right) .
$$

The quantity $\eta(S)$ is infinitesimal for all $S$ such that $t / \varepsilon S$ is limited. By Lemma 3.2 this property holds for some $S$ for which $t / \varepsilon S$ is unlimited. The real number $S$ can be chosen so that $S>1$ and $t / \varepsilon S \simeq+\infty$. Since $t$ is limited we have $\varepsilon S \simeq 0$. Then, it suffices to choose $\mu=\varepsilon$ and take $\alpha=\varepsilon S$.

Lemma 4.3. Let $\phi \in \mathcal{C}_{0}$ be standard. Let $y$ be the solution of (2.3) on $J$ with $y_{0}=\phi$, and let $T_{1}>0$ be standard such that $\left[0, T_{1}\right] \subset J$. Then there exist some positive integer $N_{0}$ and some infinitesimal partition $\left\{t_{n}: n=0, \ldots, N_{0}+1\right\}$ of $\left[0, T_{1}\right]$ such that $t_{0}=0, t_{N_{0}} \leq T_{1}<t_{N_{0}+1}, t_{n+1}=t_{n}+\alpha_{n} \simeq t_{n}$, and

$$
\frac{\varepsilon}{\alpha_{n}} \int_{t_{n} / \varepsilon}^{t_{n} / \varepsilon+\alpha_{n} / \varepsilon} f\left(\tau, y_{t_{n}}\right) d \tau \simeq f^{0}\left(y_{t_{n}}\right) .
$$

Proof. It will be done in two steps.

Step 1 . Let $t \in\left[0, T_{1}\right]$ and let us show that $y_{t}$ is nearstandard.

As $y\left(\left[-r, T_{1}\right]\right)$ is a standard compact subset of $\mathbb{R}^{d}$, it suffices to show that $y_{t}$ is $S$-continuous on $[-r, 0]$ to deduce that it is nearstandard. Taking 
into account that $\left|f^{0}(0)\right|$ is standard, for $\theta, \theta^{\prime} \in[-r, 0], \theta \leq \theta^{\prime}$, and $\theta \simeq \theta^{\prime}$, we have

$$
\begin{aligned}
\left|y_{t}\left(\theta^{\prime}\right)-y_{t}(\theta)\right| & =\left|y\left(t+\theta^{\prime}\right)-y(t+\theta)\right| \\
& \leq \int_{t+\theta}^{t+\theta^{\prime}}\left|f^{0}\left(y_{s}\right)\right| d s \\
& \leq \int_{t+\theta}^{t+\theta^{\prime}}\left|f^{0}\left(y_{s}\right)-f^{0}(0)\right| d s+\int_{t+\theta}^{t+\theta^{\prime}}\left|f^{0}(0)\right| d s \\
& \leq k \int_{t+\theta}^{t+\theta^{\prime}}|| y_{s} \| d s+\left(\theta^{\prime}-\theta\right)\left|f^{0}(0)\right| \simeq 0 .
\end{aligned}
$$

That is, $y_{t}$ is $S$-continuous on $[-r, 0]$.

Step 2. Let $\mu>0$ be given as in Lemma 4.2 , and define the set $A_{\mu}=\{\lambda \in$ $\left.\mathbb{R} / \forall t \in\left[0, T_{1}\right] \exists \alpha \in \mathbb{R}: p_{\mu}(t, \alpha, \lambda)\right\}$ where

$$
p_{\mu}(t, \alpha, \lambda) \equiv \mu<\alpha<\lambda, \quad\left|\frac{\varepsilon}{\alpha} \int_{t / \varepsilon}^{t / \varepsilon+\alpha / \varepsilon} f\left(\tau, y_{t}\right) d \tau-f^{0}\left(y_{t}\right)\right|<\lambda
$$

By Lemma 4.2 the set $A_{\mu}$ contains all the standard real numbers $\lambda>0$. By Lemma 3.3 there exists $\lambda_{0} \simeq 0$ in $A_{\mu}$, that is, there exists $0<\lambda_{0} \simeq 0$ such that for all $t \in\left[0, T_{1}\right]$ there exists $\alpha \in \mathbb{R}$ such that $D_{\mu}\left(t, \alpha, \lambda_{0}\right)$ holds. By the axiom of choice there exists a function $c:\left[0, T_{1}\right] \rightarrow \mathbb{R}$ such that $c(t)=\alpha$, that is, $p_{\mu}\left(t, c(t), \lambda_{0}\right)$ holds for all $t \in\left[0, T_{1}\right]$. Since $c(t)>\mu$ for all $t \in\left[0, T_{1}\right]$, the conclusion of the lemma is immediate.

LEMMA 4.4. Let $\phi \in \mathcal{C}_{0}$ be standard. Let $y$ be the solution of (2.3) on $J$ with $y_{0}=\phi$, and let $T_{1}>0$ be standard such that $\left[0, T_{1}\right] \subset J$. Then for all $t \in\left[0, T_{1}\right]$,

$$
\int_{0}^{t} f\left(\frac{\tau}{\varepsilon}, y_{\tau}\right) d \tau \simeq \int_{0}^{t} f^{0}\left(y_{\tau}\right) d \tau
$$

Proof. Let $f_{1}(\tau, u):=f(\tau, u)-f^{0}(u)$, for $\tau \in \mathbb{R}$ and $u \in \mathcal{C}_{0}$. The functional $f_{1}$ is Lipschitzian in $u \in \mathcal{C}_{0}$, that is, there exists some standard constant $k_{1}\left(k_{1}=2 k\right.$, where $k$ is the Lipschitz constant of $\left.f\right)$ such that

$$
\left|f_{1}\left(\tau, u_{1}\right)-f_{1}\left(\tau, u_{2}\right)\right| \leq k_{1}\left\|u_{1}-u_{2}\right\|, \quad \forall \tau \in \mathbb{R}, \forall u_{1}, u_{2} \in \mathcal{C}_{0}
$$

Next, by Lemma 4.3 there exists $\left\{t_{n}: n=0, \ldots, N_{0}+1\right\}$ such that $t_{0}=0$, $t_{N_{0}} \leq T_{1}<t_{N_{0}+1}, t_{n+1}=t_{n}+\alpha_{n} \simeq t_{n}$, and

$$
\frac{\varepsilon}{\alpha_{n}} \int_{t_{n} / \varepsilon}^{t_{n} / \varepsilon+\alpha_{n} / \varepsilon} f_{1}\left(\tau, y_{t_{n}}\right) d \tau \simeq 0
$$


10 Time averaging for functional differential equations

Let $t \in\left[0, T_{1}\right]$ and let $N$ be a positive integer such that $t_{N} \leq t<t_{N+1}$. We have

$$
\begin{aligned}
\left|\int_{t_{N}}^{t} f_{1}\left(\frac{\tau}{\varepsilon}, y_{\tau}\right) d \tau\right| \leq & \left|\int_{t_{N}}^{t} f_{1}\left(\frac{\tau}{\varepsilon}, y_{\tau}\right) d \tau-\int_{t_{N}}^{t} f_{1}\left(\frac{\tau}{\varepsilon}, 0\right) d \tau\right| \\
& +\left|\int_{t_{N}}^{t} f_{1}\left(\frac{\tau}{\varepsilon}, 0\right) d \tau\right| \\
& \leq \int_{t_{N}}^{t}\left|f_{1}\left(\frac{\tau}{\varepsilon}, y_{\tau}\right)-f_{1}\left(\frac{\tau}{\varepsilon}, 0\right)\right| d \tau+\left|\int_{t_{N}}^{t} f_{1}\left(\frac{\tau}{\varepsilon}, 0\right) d \tau\right| \\
& \leq k_{1} \int_{t_{N}}^{t}\left\|y_{\tau}\right\| d \tau+\left|\int_{t_{N}}^{t} f_{1}\left(\frac{\tau}{\varepsilon}, 0\right) d \tau\right| .
\end{aligned}
$$

As $y\left(\left[-r, T_{1}\right]\right)$ is a standard compact subset of $\mathbb{R}^{d}$, it follows that

$$
\int_{t_{N}}^{t}\left\|y_{\tau}\right\| d \tau \simeq 0
$$

We now estimate the second term in the right-hand side of (4.21). For this, consider all the cases.

Case 1 (Both $t_{N} / \varepsilon$ and $t / \varepsilon$ are limited). In this case, it is clear that

$$
\left|\int_{t_{N}}^{t} f_{1}\left(\frac{\tau}{\varepsilon}, 0\right) d \tau\right|=\varepsilon\left|\int_{t_{N} / \varepsilon}^{t / \varepsilon} f_{1}(s, 0) d s\right| \simeq 0 .
$$

Case 2 (Both $t_{N} / \varepsilon$ and $t / \varepsilon$ are unlimited). By means of condition $\left(\mathrm{H}^{\prime}\right)$, we have

$$
\begin{aligned}
\left|\int_{t_{N}}^{t} f_{1}\left(\frac{\tau}{\varepsilon}, 0\right) d \tau\right| & =\varepsilon\left|\int_{t_{N} / \varepsilon}^{t / \varepsilon} f_{1}(s, 0) d s\right| \\
& \leq t_{N}\left|\frac{1}{t_{N} / \varepsilon} \int_{0}^{t_{N} / \varepsilon} f_{1}(s, 0) d s\right|+t\left|\frac{1}{t / \varepsilon} \int_{0}^{t / \varepsilon} f_{1}(s, 0) d s\right| \simeq 0 .
\end{aligned}
$$


Case $3\left(t_{N} / \varepsilon\right.$ is limited and $t / \varepsilon$ is unlimited). This case is a combination of Cases 1 and 2. We write

$$
\begin{aligned}
\left|\int_{t_{N}}^{t} f_{1}\left(\frac{\tau}{\varepsilon}, 0\right) d \tau\right| & =\varepsilon\left|\int_{t_{N} / \varepsilon}^{t / \varepsilon} f_{1}(s, 0) d s\right| \\
& \leq \varepsilon\left|\int_{0}^{t_{N} / \varepsilon} f_{1}(s, 0) d s\right|+t\left|\frac{1}{t / \varepsilon} \int_{0}^{t / \varepsilon} f_{1}(s, 0) d s\right| \\
& \simeq 0
\end{aligned}
$$

Thus, we have

$$
\left|\int_{t_{N}}^{t} f_{1}\left(\frac{\tau}{\varepsilon}, 0\right) d \tau\right| \simeq 0 .
$$

Therefore, from (4.21) and by means of (4.22) and (4.26), we obtain that

$$
\left|\int_{t_{N}}^{t} f_{1}\left(\frac{\tau}{\varepsilon}, y_{\tau}\right) d \tau\right| \simeq 0
$$

so that

$$
\begin{aligned}
\int_{0}^{t} f( & \left.\frac{\tau}{\varepsilon}, y_{\tau}\right) d \tau-\int_{0}^{t} f^{0}\left(y_{\tau}\right) d \tau \\
= & \int_{0}^{t} f_{1}\left(\frac{\tau}{\varepsilon}, y_{\tau}\right) d \tau \\
\simeq & \sum_{n=0}^{N-1} \int_{t_{n}}^{t_{n+1}} f_{1}\left(\frac{\tau}{\varepsilon}, y_{\tau}\right) d \tau \\
= & \sum_{n=0}^{N-1} \int_{t_{n}}^{t_{n+1}}\left(f_{1}\left(\frac{\tau}{\varepsilon}, y_{\tau}\right)-f_{1}\left(\frac{\tau}{\varepsilon}, y_{t_{n}}\right)\right) d \tau \\
& +\sum_{n=0}^{N-1} \int_{t_{n}}^{t_{n+1}} f_{1}\left(\frac{\tau}{\varepsilon}, y_{t_{n}}\right) d \tau .
\end{aligned}
$$

Let $\tau \in\left[t_{n}, t_{n+1}\right], n=0, \ldots, N$, and let $\theta \in[-r, 0]$. We have

$$
\begin{aligned}
\left|y_{\tau}(\theta)-y_{t_{n}}(\theta)\right| & =\left|y(\tau+\theta)-y\left(t_{n}+\theta\right)\right| \\
& \leq \int_{t_{n}+\theta}^{\tau+\theta}\left|f^{0}\left(y_{s}\right)\right| d s
\end{aligned}
$$


12 Time averaging for functional differential equations

$$
\begin{aligned}
& \leq \int_{t_{n}+\theta}^{\tau+\theta}\left|f^{0}\left(y_{s}\right)-f^{0}(0)\right| d s+\int_{t_{n}+\theta}^{\tau+\theta}\left|f^{0}(0)\right| d s \\
& \leq k \int_{t_{n}+\theta}^{\tau+\theta}\left\|y_{s}\right\| d s+\left(\tau-t_{n}\right)\left|f^{0}(0)\right| .
\end{aligned}
$$

As $y\left(\left[-r, T_{1}\right]\right)$ is a standard compact subset of $\mathbb{R}^{d}$ and $\left|f^{0}(0)\right|$ is standard, from (4.29) we deduce that

$$
\left|y_{\tau}(\theta)-y_{t_{n}}(\theta)\right| \simeq 0
$$

That is, $y_{\tau} \simeq y_{t_{n}}$ for $\tau \in\left[t_{n}, t_{n+1}\right], n=0, \ldots, N$. By Lemma 3.4, we have

$$
\sup \left\{\left\|y_{\tau}-y_{t_{n}}\right\|: \tau \in\left[t_{n}, t_{n+1}\right], 0 \leq n \leq N-1\right\} \simeq 0
$$

and so is

$$
k_{1} \cdot \sup \left\{\left\|y_{\tau}-y_{t_{n}}\right\|: \tau \in\left[t_{n}, t_{n+1}\right], 0 \leq n \leq N-1\right\} \cdot t_{N},
$$

so that

$$
\begin{aligned}
& \left|\sum_{n=0}^{N-1} \int_{t_{n}}^{t_{n+1}} f_{1}\left(\frac{\tau}{\varepsilon}, y_{\tau}\right)-f_{1}\left(\frac{\tau}{\varepsilon}, y_{t_{n}}\right) d \tau\right| \\
& \quad \leq \sum_{n=0}^{N-1} \int_{t_{n}}^{t_{n+1}}\left|f_{1}\left(\frac{\tau}{\varepsilon}, y_{\tau}\right)-f_{1}\left(\frac{\tau}{\varepsilon}, y_{t_{n}}\right)\right| d \tau \\
& \quad \leq k_{1} \sum_{n=0}^{N-1} \int_{t_{n}}^{t_{n+1}}\left\|y_{\tau}-y_{t_{n}}\right\| d \tau \\
& \quad \leq k_{1} \cdot \sup \left\{\left\|y_{\tau}-y_{t_{n}}\right\|: \tau \in\left[t_{n}, t_{n+1}\right], 0 \leq n \leq N-1\right\} \cdot t_{N} \\
& \quad \simeq 0 .
\end{aligned}
$$


Therefore, from (4.28) and by means of (4.20), it follows that

$$
\begin{aligned}
\int_{0}^{t} f\left(\frac{\tau}{\varepsilon}, y_{\tau}\right) d \tau-\int_{0}^{t} f^{0}\left(y_{\tau}\right) d \tau \\
\simeq \sum_{n=0}^{N-1} \int_{t_{n}}^{t_{n+1}} f_{1}\left(\frac{\tau}{\varepsilon}, y_{t_{n}}\right) d \tau \\
=\sum_{n=0}^{N-1} \int_{t_{n}}^{t_{n}+\alpha_{n}} f_{1}\left(\frac{\tau}{\varepsilon}, y_{t_{n}}\right) d \tau \\
=\varepsilon \sum_{n=0}^{N-1} \int_{t_{n} / \varepsilon}^{t_{n} / \varepsilon+\alpha_{n} / \varepsilon} f_{1}\left(\tau, y_{t_{n}}\right) d \tau \\
=\sum_{n=0}^{N-1} \alpha_{n}\left(\frac{\varepsilon}{\alpha_{n}} \int_{t_{n} / \varepsilon}^{t_{n} / \varepsilon+\alpha_{n} / \varepsilon} f_{1}\left(\tau, y_{t_{n}}\right) d \tau\right) \\
=\sum_{n=0}^{N-1} \alpha_{n} \beta_{n} \\
\simeq 0
\end{aligned}
$$

since $\left|\sum_{n=0}^{N-1} \alpha_{n} \cdot \beta_{n}\right| \leq \bar{\beta} \sum_{n=0}^{N-1} \alpha_{n}=\bar{\beta} \sum_{n=0}^{N-1}\left(t_{n+1}-t_{n}\right)=\bar{\beta} \cdot t_{N}$, where $\bar{\beta}=$ $\max \left\{\left|\beta_{n}\right|: 0 \leq n \leq N-1\right\}$. By Lemma 3.4, $\bar{\beta}$ is infinitesimal and so is $\bar{\beta} \cdot t_{N}$. This completes the proof of Lemma 4.4.

Lemma 4.5. Let $\phi \in \mathcal{C}_{0}$ be standard. Let $x$ be the solution of (1.1) on I, and $y$ the solution of (2.3) on J, with $x_{0}=y_{0}=\phi$. Let $T_{1}>0$ be standard such that $\left[0, T_{1}\right] \subset I \cap J$. Then $x(t) \simeq y(t)$ for all $t \in\left[0, T_{1}\right]$.

Proof. For $t \in\left[0, T_{1}\right]$, we have

$$
\begin{aligned}
& x(t)=\phi(0)+\int_{0}^{t} f\left(\frac{\tau}{\varepsilon}, x_{\tau}\right) d \tau, \\
& y(t)=\phi(0)+\int_{0}^{t} f^{0}\left(y_{\tau}\right) d \tau .
\end{aligned}
$$

Subtraction of (4.35) and (4.36) gives

$$
\begin{aligned}
|x(t)-y(t)| \leq & \left|\int_{0}^{t}\left(f\left(\frac{\tau}{\varepsilon}, x_{\tau}\right)-f\left(\frac{\tau}{\varepsilon}, y_{\tau}\right)\right) d \tau\right| \\
& +\left|\int_{0}^{t}\left(f\left(\frac{\tau}{\varepsilon}, y_{\tau}\right)-f^{0}\left(y_{\tau}\right)\right) d \tau\right|
\end{aligned}
$$


14 Time averaging for functional differential equations

$$
\begin{aligned}
\leq & \int_{0}^{t}\left|f\left(\frac{\tau}{\varepsilon}, x_{\tau}\right)-f\left(\frac{\tau}{\varepsilon}, y_{\tau}\right)\right| d \tau \\
& +\left|\int_{0}^{t}\left(f\left(\frac{\tau}{\varepsilon}, y_{\tau}\right)-f^{0}\left(y_{\tau}\right)\right) d \tau\right| \\
\leq & k \int_{0}^{t}\left\|x_{\tau}-y_{\tau}\right\| d \tau+\left|\int_{0}^{t}\left(f\left(\frac{\tau}{\varepsilon}, y_{\tau}\right)-f^{0}\left(y_{\tau}\right)\right) d \tau\right| .
\end{aligned}
$$

Since, for $\tau \in[0, t],\left\|x_{\tau}-y_{\tau}\right\| \leq \sup _{s \in[0, \tau]}|x(s)-y(s)|$, it follows from (4.37) that

$$
\begin{aligned}
|x(t)-y(t)| \leq & k \int_{0}^{t} \sup _{s \in[0, \tau]}|x(s)-y(s)| d \tau \\
& +\left|\int_{0}^{t}\left(f\left(\frac{\tau}{\varepsilon}, y_{\tau}\right)-f^{0}\left(y_{\tau}\right)\right) d \tau\right| .
\end{aligned}
$$

The first term on the right-hand side of (4.38) is increasing and therefore

$$
\begin{aligned}
\sup _{\tau \in[0, t]}|x(\tau)-y(\tau)| \leq & k \int_{0}^{t} \sup _{s \in[0, \tau]}|x(s)-y(s)| d \tau \\
& +\sup _{\tau \in[0, t]}\left|\int_{0}^{\tau}\left(f\left(\frac{s}{\varepsilon}, y_{s}\right)-f^{0}\left(y_{s}\right)\right) d s\right| .
\end{aligned}
$$

By Gronwall's lemma, this implies that

$$
\sup _{\tau \in[0, t]}|x(\tau)-y(\tau)| \leq e^{k t} \sup _{\tau \in[0, t]}\left|\int_{0}^{\tau}\left(f\left(\frac{s}{\varepsilon}, y_{s}\right)-f^{0}\left(y_{s}\right)\right) d s\right|
$$

and by means of Lemmas 4.4 and 3.4, respectively, we conclude that $x(t) \simeq y(t)$, which finishes the proof.

\subsection{Proof of Theorem 3.6}

For notation simplicity, we let $t_{0}=0$. Let $T>0$ be standard in $J$. Let $K$ be a standard tubular neighborhood of diameter $\rho$ around $\Gamma=y([0, T])$. Let $I$ be the maximal interval of definition of $x$. Define the set $A=\left\{T_{1} \in\right.$ $\left.I \cap[0, T] / x\left(\left[0, T_{1}\right]\right) \subset K\right\}$. The set $A$ is nonempty $(0 \in A)$ and bounded above by $T$. Let $T_{0}$ be a lower upper bound of $A$. There is $T_{1} \in A$ such that $T_{0}-\varepsilon^{2}<T_{1} \leq T_{0}$. By continuation, there is $T_{2}, T_{2}$ appreciable, such that $x$ remains defined on $\left[0, T_{1}+\varepsilon T_{2}\right]$. Likewise, by continuation, $y$ remains defined in particular on the same interval. By Lemma 4.5, we have $x(t) \simeq$ $y(t)$ for $t \in\left[0, T_{1}+\varepsilon T_{2}\right]$. Suppose $T_{1}+\varepsilon T_{2} \leq T$. Then, $\left[0, T_{1}+\varepsilon T_{2}\right] \subset I$ and 
$x\left(\left[0, T_{1}+\varepsilon T_{2}\right]\right) \subset K$ imply that $T_{1}+\varepsilon T_{2} \in A$, which is a contradiction with $T_{1}+\varepsilon T_{2}>T_{0}$. Thus $T_{1}+\varepsilon T_{2}>T$, that is, we have $x(t) \simeq y(t)$ for all $t \in$ $[0, T] \subset\left[0, T_{1}+\varepsilon T_{2}\right]$.

\section{Acknowledgment}

The author is very grateful to the anonymous referee for suggesting some improvements in the presentation of this paper.

\section{References}

[1] N. N. Bogoliubov and Y. A. Mitropolsky, Asymptotic Methods in the Theory of Non-Linear Oscillations, International Monographs on Advanced Mathematics and Physics, Gordon and Breach Science Publishers, New York, 1961.

[2] F. Diener and M. Diener (eds.), Nonstandard Analysis in Practice, Universitext, Springer-Verlag, Berlin, 1995.

[3] M. Diener and C. Lobry (eds.), Actes de l'École d'Été: Analyse Non Standard et Représentation du Réel [Proceedings of the Summer School on Nonstandard Analysis and Representation of the Real ], Office des Publications Universitaires, Algiers, 1985 (French).

[4] M. Diener and G. Wallet (eds.), Mathématiques Finitaires \& Analyse Non Standard. Tome 1, 2 [Finitary Mathematics and Nonstandard Analysis. Vol. 1, 2], Publications Mathématiques de l'Université Paris VII, vol. 31, Université de Paris VII U.E.R. de Mathématiques, Paris, 1989 (French).

[5] J. Guckenheimer and P. Holmes, Nonlinear Oscillations, Dynamical Systems, and Bifurcations of Vector Fields, Applied Mathematical Sciences, vol. 42, Springer-Verlag, New York, 1983.

[6] A. Halanay, On the method of averaging for differential equations with retarded argument, J. Math. Anal. Appl. 14 (1966), 70-76.

[7] J. K. Hale, Averaging methods for differential equations with retarded arguments and a small parameter, J. Differential Equations 2 (1966), 57-73.

[8] _ Ordinary Differential Equations, Pure and Applied Mathematics, vol. 21, Wiley-Interscience, New York, 1969.

[9] J. K. Hale and S. M. Verduyn Lunel, Averaging in infinite dimensions, J. Integral Equations Appl. 2 (1990), no. 4, 463-494.

[10] Introduction to Functional-Differential Equations, Applied Mathematical Sciences, vol. 99, Springer-Verlag, New York, 1993.

[11] T. Janiak and E. Łuczak-Kumorek, A theorem on partial middling for functionaldifferential equations of the neutral type, Univ. $\mathrm{u}$ Novom Sadu $\mathrm{Zb}$. Rad. Prirod.-Mat. Fak. Ser. Mat. 16 (1986), no. 2, 107-119.

[12] - The theorem of middling for functional-differential equations of neutral type, Discuss. Math. 11 (1991), 63-73.

[13] M. Lakrib, Sur la moyennisation dans les systèmes à plusieurs fréquences, to appear in Maghreb Math. Rev.

[14] - On the validity of the averaging method for all time, Maghreb Math. Rev. 8 (1999), no. 1-2, 119-128. 
16 Time averaging for functional differential equations

[15] _ The method of averaging and functional differential equations with delay, Int. J. Math. Math. Sci. 26 (2001), no. 8, 497-511.

[16] M. Lakrib and T. Sari, Averaging method for functional differential equations, submitted.

[17] R. Lutz and M. Goze, Nonstandard Analysis. A Practical Guide with Applications, Lecture Notes in Mathematics, vol. 881, Springer-Verlag, Berlin, 1981.

[18] R. Lutz and T. Sari, Applications of nonstandard analysis to boundary value problems in singular perturbation theory, Theory and Applications of Singular Perturbations (Oberwolfach, 1981), Lecture Notes in Mathematics, vol. 942, Springer, Berlin, 1982, pp. 113-135.

[19] G. N. Medvedev, Asymptotic solutions of some systems of differential equations with deviating argument, Soviet Math. Dokl. 9 (1968), 85-87.

[20] E. Nelson, Internal set theory: a new approach to nonstandard analysis, Bull. Amer. Math. Soc. 83 (1977), no. 6, 1165-1198.

[21] A. Robinson, Nonstandard Analysis, American Elsevier, New York, 1974.

[22] J. A. Sanders and F. Verhulst, Averaging Methods in Nonlinear Dynamical Systems, Applied Mathematical Sciences, vol. 59, Springer-Verlag, New York, 1985.

[23] T. Sari, Nonstandard perturbation theory of differential equations, presented as an invited talk at the International Research Symposium on Nonstandard Analysis and Its Applications, ICMS, Edinburgh, 11-17 August 1996.

[24] Stroboscopy and averaging, Colloque Trajectorien à la Mémoire de Georges Reeb et Jean-Louis Callot (Strasbourg-Obernai, 1995) (A. Fruchard and A. Troesch, eds.), Prépublication de l'Institut de Recherche Mathematique Avancee, Université Louis Pasteur, Strasbourg, 1995, pp. 95-124.

Mustapha Lakrib: Laboratoire de Mathématiques, Université de Haute Alsace, 4 rue des Frères Lumière, 68093 Mulhouse, France

E-mail address: M.Lakrib@uha.fr 


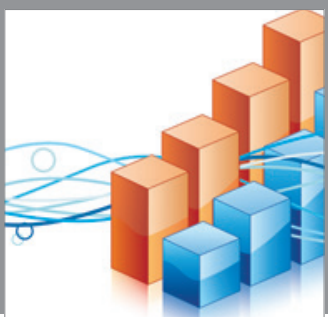

Advances in

Operations Research

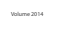

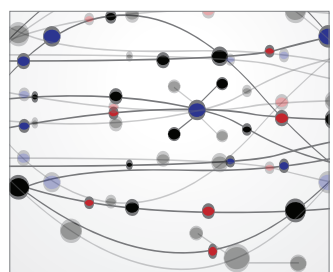

\section{The Scientific} World Journal
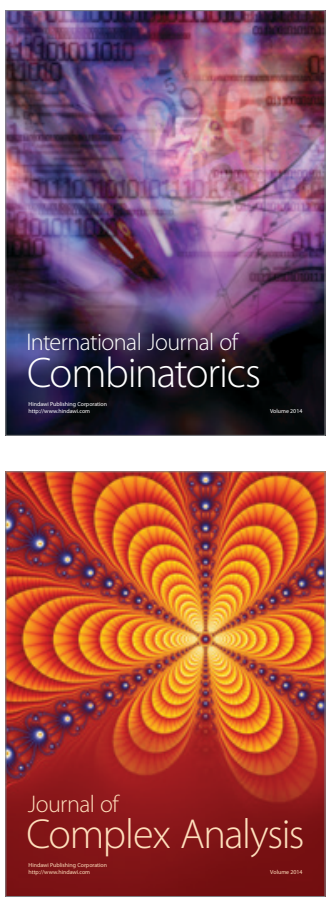

International Journal of

Mathematics and

Mathematical

Sciences
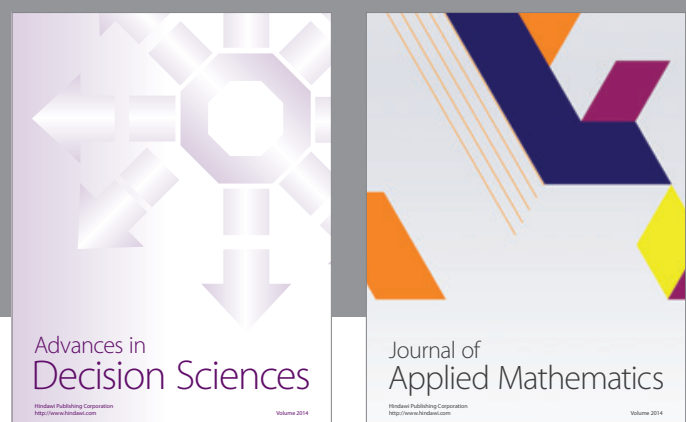

Journal of

Applied Mathematics
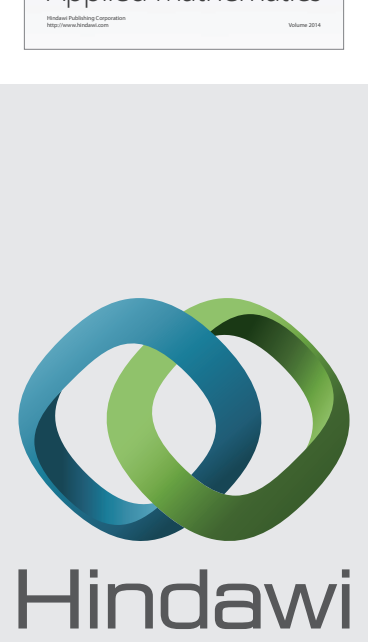

Submit your manuscripts at http://www.hindawi.com
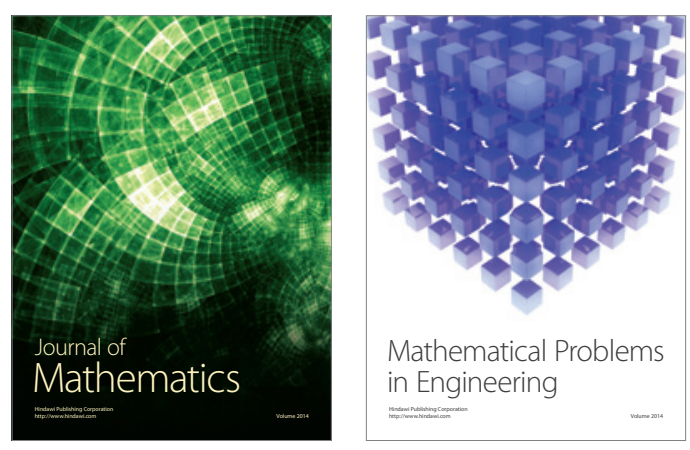

Mathematical Problems in Engineering
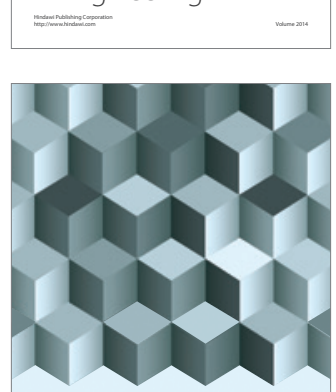

Journal of

Function Spaces
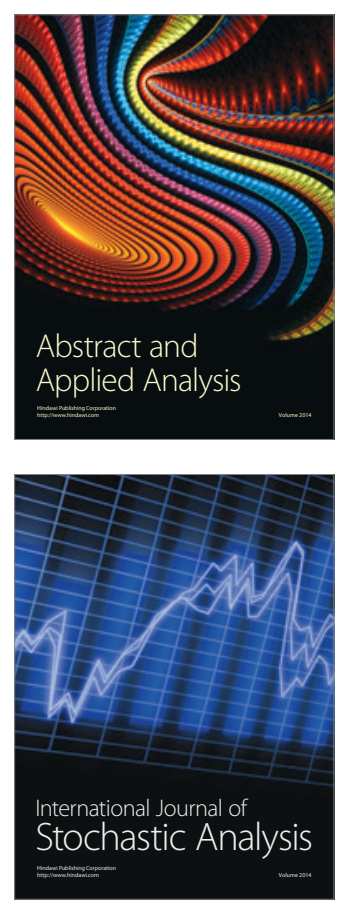

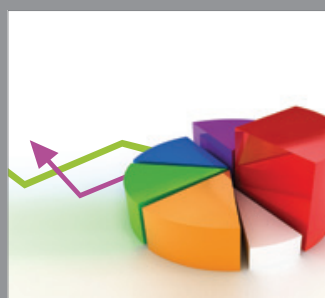

ournal of

Probability and Statistics

Promensencen
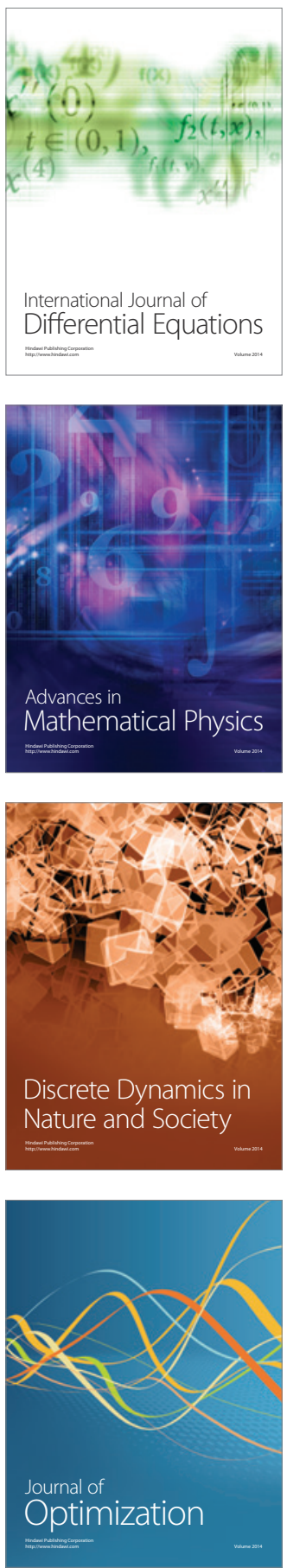